\title{
Screw Performance Degradation Assessment Based on Quantum Genetic Algorithm and Dynamic Fuzzy Neural Network
}

\author{
Xiaochen Zhang, Hongli Gao, and Haifeng Huang \\ School of Mechanical Engineering, Southwest Jiaotong University, Chengdu 610031, China \\ Correspondence should be addressed to Hongli Gao; ghl2248@hotmail.com
}

Received 16 April 2015; Revised 23 June 2015; Accepted 1 July 2015

Academic Editor: Wahyu Caesarendra

Copyright (C) 2015 Xiaochen Zhang et al. This is an open access article distributed under the Creative Commons Attribution License, which permits unrestricted use, distribution, and reproduction in any medium, provided the original work is properly cited.

To evaluate the performance of ball screw, screw performance degradation assessment technology based on quantum genetic algorithm (QGA) and dynamic fuzzy neural network (DFNN) is studied. The ball screw of the CINCINNATIV5-3000 machining center is treated as the study object. Two Kistler 8704B100M1 accelerometers and a Kistler 8765A250M5 three-way accelerometer are installed to monitor the degradation trend of screw performance. First, screw vibration signal features are extracted both in time domain and frequency domain. Then the feature vectors can be obtained by principal component analysis (PCA). Second, the initialization parameters of the DFNN are optimized by means of QGA. Finally, the feature vectors are inputted to DFNN for training and then get the screw performance degradation model. The experiment results show that the screw performance degradation model could effectively evaluate the performance of NC machine screw.

\section{Introduction}

NC machine tool is an important foundation for modern manufacturing, which directly impacts the economic development $[1,2]$. With the continuous development of modern manufacturing technology, NC machine tool has been widely used in aerospace, automobile, shipbuilding, and other industries $[3,4]$. In the manufacturing process, the continuous degradation of ball screw directly leads to the decrease of NC machine tool's static geometric accuracy and dynamic precision, which means that enterprises should increase their production and maintenance costs. Since ball screw's performance would change greatly with the variation of working conditions and processing environment, replacement of the ball screw regularly is neither scientific nor reasonable $[5,6]$. Therefore, screw performance degradation needs real-time assessment in order to cut down enterprises' maintenance costs.

DFNN is a kind of information processing method combined with fuzzy set theory. Its essence is a dynamic mapping network with fuzzy input signal and weights [7].
During the learning process, network parameters and dimension will change according to the rules. Compared with the traditional neural network, DFNN is more suitable for describing dynamic system. However, it is difficult to choose the initialization parameters of the DFNN, which means DFNN will easily fall into local optimum $[8,9]$. To the defect of DFNN, this paper adopts the QGA, which has the advantage of the high efficiency and avoiding local optimum, to select the best initialization parameters of the DFNN that can improve the performance of DFNN and increase the operation stability of system.

The purpose of this paper is to present a useful method for performance degradation assessment of ball screw based on QGA and DFNN. Meanwhile feature vectors selection method is proposed here. Particularly, we extract screw vibration signal features both in time domain and frequency domain. The dimensionalities of the input signal feature space are reduced with the help of PCA. Then the initialization parameters of the DFNN are optimized by means of QGA. Screw performance degradation model can be gotten after training with the feature vectors. Finally, we compare 
the prediction accuracies among different kinds of neural networks to examine the effectiveness of the proposed method.

\section{Screw Vibration Feature Vectors}

2.1. Screw Online Monitoring System. Figure 1 shows the screw online monitoring system applied to CINCINNATIV53000 machining center. Two Kistler 8704B100M1 one-way accelerometers are installed at two bearing chocks to monitor the radial vibration of screw ends, while a Kistler 8765A250M5 three-way accelerometer is installed on the screw nut to monitor three-dimensional shaking of the screw nut. INV1870 is the signal conditioner connected with Advantech PCI1710 data acquisition card. Industry computer can store the vibration data through the PCI1710 data acquisition card, and the sampling frequency is $2.56 \mathrm{kHz}$. Ball screw works in horizontal installation state; meanwhile the installation method of the ball screw is one-end fixed while the other end floated. Ball screw reaches $45 \mathrm{~mm}$ in axis diameter, $12 \mathrm{~mm}$ in lead, and $762 \mathrm{~mm}$ in journey.

2.2. Signal Analysis and Feature Extraction. With the increase of service life, ball screw's performance will gradually reduce; meanwhile the vibration of the screw fixed end changes gradually. After more than five years of service life, the vibration increased significantly. Failure occurs at the seventh year of service life. Vibrations of screw fixed end under different service life are showed in Figure 2.

Considering the distribution characteristics of the ball screw vibration signal, the original features consist of the following parts. Time domain or frequency domain features (such as root mean square value, peak value, and gravity frequency), which are presently used to reflect the time domain or frequency domain, are extracted as a part of the original signal features. By using the wavelet analysis method, the vibration signal is decomposed into 5 levels selecting wavelet "dbl," and distinct time-frequency features based on wavelet packet energy are obtained. Approximate entropy, a recently developed statistic theory in mechanical fault diagnosis, has also been applied to enrich the original features here.

The original features extracted by different methods, which have characteristics of high dimension and heavy computation, are not conducive to the online modeling and evaluation. Meanwhile, considering the certain correlation among the high dimensional original features, eliminating redundant information is one of the main focuses in the research of feature extraction.

PCA is a statistical method that uses an orthogonal transformation to convert a set of observations of possibly correlated variables into a set of values of linearly uncorrelated variables. The $n$-dimensional original features can be expressed as $\mathbf{X}=\left[x_{1}, x_{2}, \ldots, x_{n}\right]$. By means of PCA, linearly uncorrelated feature vectors, $\mathbf{Y}=\left[y_{1}, y_{2}, \ldots, y_{n}\right]$, can be obtained. The contribution of the $i$ th component $\eta_{i}$ can be expressed as follows:

$$
\eta_{i}=\frac{\lambda_{i}}{\sum_{k=1}^{n} \lambda_{k}}
$$

where $\lambda_{i}$ is the variance of the $y_{i}$.
Then the contributions of the first $m$ principal component $\eta_{m}^{\prime}$ can be built as follows:

$$
\eta_{m}^{\prime}=\frac{\sum_{i=1}^{m} \lambda_{i}}{\sum_{k=1}^{n} \lambda_{k}}
$$

The first ten principal component contributions are plotted, as shown in Figure 3. The first three principal component contributions are more than 20\%, and the accumulative contribution of the first five principal components is over $90 \%$. Taking into account the real-time operation speed and operation precision, this paper takes the first five principal components as the feature vectors of the screw performance assessment system.

\section{Screw Performance Degradation Assessment}

3.1. Dynamic Fuzzy Neural Network. The DFNN has five layers, including input layer, output layer, and three hidden layers $[10,11]$. Figure 4 shows the structure of DFNN. In Figure $4, x_{1}, x_{2}, \ldots, x_{r}$ are the input variables, $y$ is the system output, $\mathrm{MF}_{i j}$ is the $j$ th membership function of the $i$ th input variable, $N_{j}$ is the $j$ th normalized node, and $w_{j}$ is the weight of the $j$ th rule. The number of the system rules is $u$.

The main function of each layer can be explained as follows.

(1) Input layer: The variables $x_{1}, x_{2}, \ldots, x_{r}$ are input to the corresponding nodes, respectively.

(2) Membership function layer: Each node represents a membership function, which can be expressed as follows:

$$
\mu_{i j}\left(x_{i}\right)=\exp \left[-\frac{\left(x_{i}-c_{i j}\right)^{2}}{\sigma_{j}^{2}}\right],
$$

where $\mu_{i j}(i=1,2, \ldots, r ; j=1,2, \ldots, u)$ is the $j$ th membership function of the $x_{i} \cdot c_{i j}$ and $\sigma_{j}$ represent the center and width of the $\mu_{i j}$, respectively.

(3) $t$-norm layer: In the layer, fuzzy rules are represented by nodes. The output of the $j$ th node $R_{j}$ is defined as

$$
\begin{array}{r}
\phi_{j}=\exp \left[-\frac{\sum_{i=1}^{r}\left(x_{i}-c_{i j}\right)^{2}}{\sigma_{j}^{2}}\right]=\exp \left[-\frac{\|\mathbf{X}-\mathbf{C}\|^{2}}{\sigma_{j}^{2}}\right], \\
j=1,2, \ldots, u .
\end{array}
$$

(4) Normalized layer: Normalization processing is made for the output of the $t$-norm layer in this layer. The output of $j$ th node $N_{j}$ can be expressed as follows:

$$
\psi_{j}=\frac{\phi_{j}}{\sum_{k=1}^{u} \phi_{k}}, \quad j=1,2, \ldots, u .
$$

(5) Output layer: The system output which is the superposition of the input variables can be formulated as

$$
y(\mathbf{X})=\sum_{k=1}^{u} w_{k} \cdot \psi_{k} .
$$




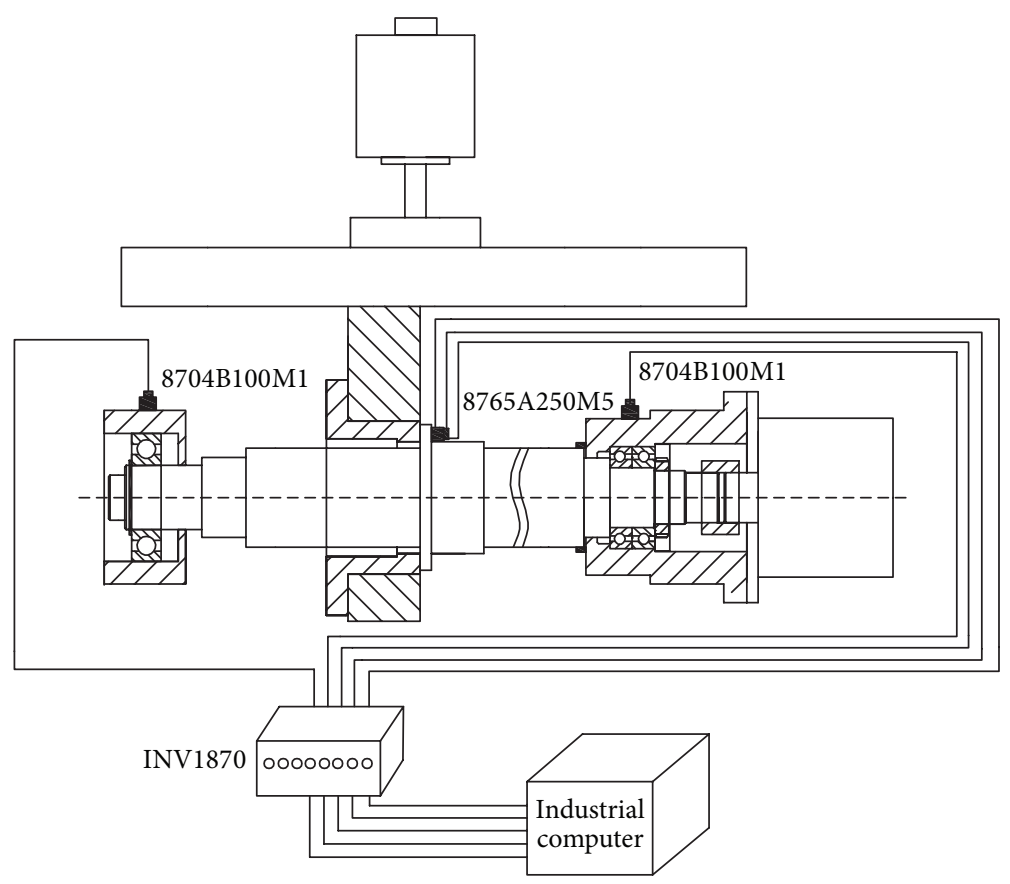

FIGURE 1: Screw online monitoring system.

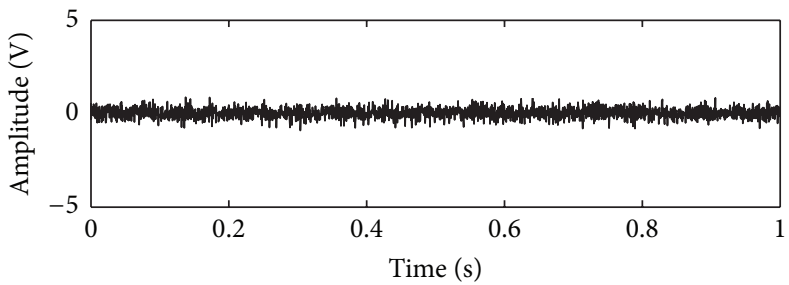

(a)

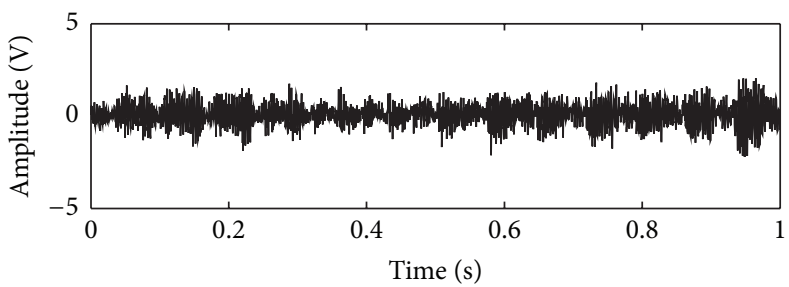

(c)

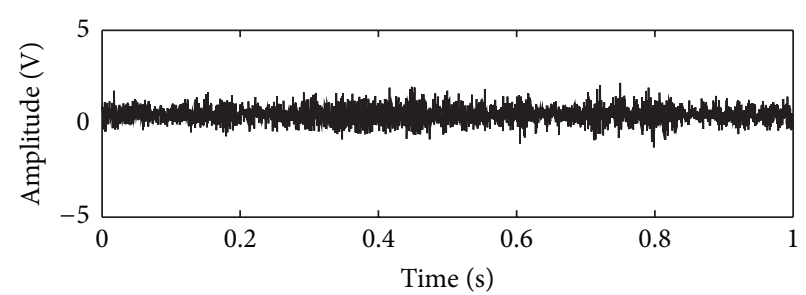

(b)

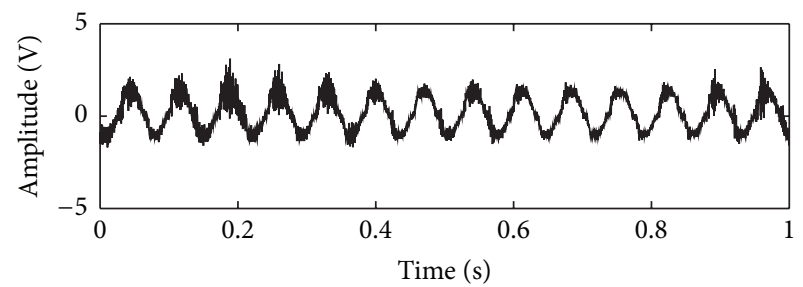

(d)

FIGURE 2: Vibrations of screw fixed end under different service life: (a) vibration of 0.5-year-service screw, (b) vibration of 2-year-service screw, (c) vibration of 5-year-service screw, and (d) vibration of 7-year-service screw.

Before generating the first rule, DFNN should set the initialization parameters of the network. Ten parameters of DFNN need to be initialized, including membership function width of the first rule $\sigma_{0}$, overlap factor of the radial based function $k$, width renewal factor $k_{w}$, rule threshold $k_{\text {err }}$, decay constant $\gamma$, convergence constant $\beta$, maximum debugging standard $\varepsilon_{\max }$, minimum debugging standard $\varepsilon_{\min }$, maximum output error $e_{\max }$, and minimum output error $e_{\min }$. The specific details of the ten initialization parameters are introduced in [12-15].
DFNN will easily fall into local optimum due to the random initialization of the network parameters. Therefore, this paper applies QGA to obtain the optimal initialization parameters of DFNN.

3.2. Quantum Genetic Algorithm. The main ideas of QGA can be expressed as follows: according to the parameter characteristics of DFNN, chromosomal genes with quantum bit coding system are constructed and the population that includes several chromosomes is generated. By adopting 


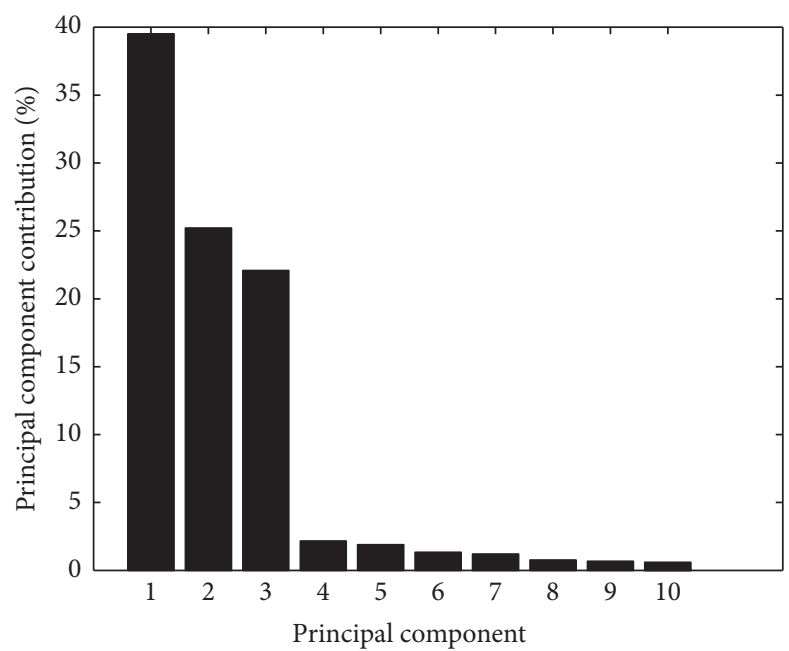

FIgURE 3: The first ten principal component contributions.

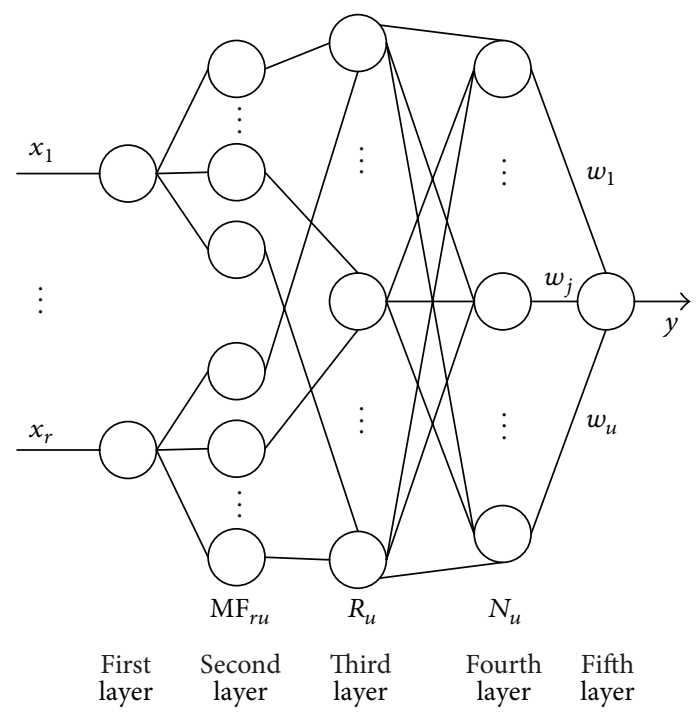

FIGURE 4: The structure of DFNN.

quantum cross, variation, and quantum rotation gate, the optimal initialization parameters of DFNN can be obtained. Figure 5 shows the algorithm flowchart of QGA.
3.2.1. Population Initialization. The population size of the initialized population $\mathbf{Q}\left(t_{0}\right)=\left\{\mathbf{q}_{1}^{t_{0}}, \mathbf{q}_{2}^{t_{0}}, \ldots, \mathbf{q}_{N}^{t_{0}}\right\}$ is $N$. By means of the quantum bit coding system, arbitrary chromosome $\mathbf{q}_{j}^{t_{0}}$ of the initialized population $\mathbf{Q}\left(t_{0}\right)$ can be expressed as follows:

$$
\mathbf{q}_{j}^{t_{0}}=\left[\begin{array}{ccccccccccccc}
\alpha_{11}^{t_{0}} & \alpha_{12}^{t_{0}} & \cdots & \alpha_{1 k_{1}}^{t_{0}} & \alpha_{21}^{t_{0}} & \alpha_{22}^{t_{0}} & \cdots & \alpha_{2 k_{2}}^{t_{0}} & \cdots & \alpha_{m 1}^{t_{0}} & \alpha_{m 2}^{t_{0}} & \cdots & \alpha_{m k_{m}}^{t_{0}} \\
\beta_{11}^{t_{0}} & \beta_{12}^{t_{0}} & \cdots & \beta_{1 k_{1}}^{t_{0}} & \beta_{21}^{t_{0}} & \beta_{22}^{t_{0}} & \cdots & \beta_{2 k_{2}}^{t_{0}} & \cdots & \beta_{m 1}^{t_{0}} & \beta_{m 2}^{t_{0}} & \cdots & \beta_{m k_{m}}^{t_{0}}
\end{array}\right],
$$

where $m$ is the number of the chromosomal genes and $k_{1}, k_{2}, \ldots, k_{m}$ represent the quantum bit number of each chromosomal gene, respectively. $\mathbf{q}_{j}^{t_{0}}$ is the $j$ th chromosome of the $t_{0}$ generation. In this paper, chromosome $\mathbf{q}_{j}^{t_{0}}$ includes ten genes, which are, respectively, corresponding to ten initialization parameters of DFNN. Initialization probability amplitude $[\alpha, \beta]^{T}$ is $[1 / \sqrt{2}, 1 / \sqrt{2}]^{T}$ so that each chromosome expresses the same state. The fitness function is built as follows $[16,17]$ :

$$
f=1-\frac{1}{s} \sum_{p=1}^{s}\left|\frac{y_{p}^{\prime}-t_{p}}{t_{p}}\right|
$$




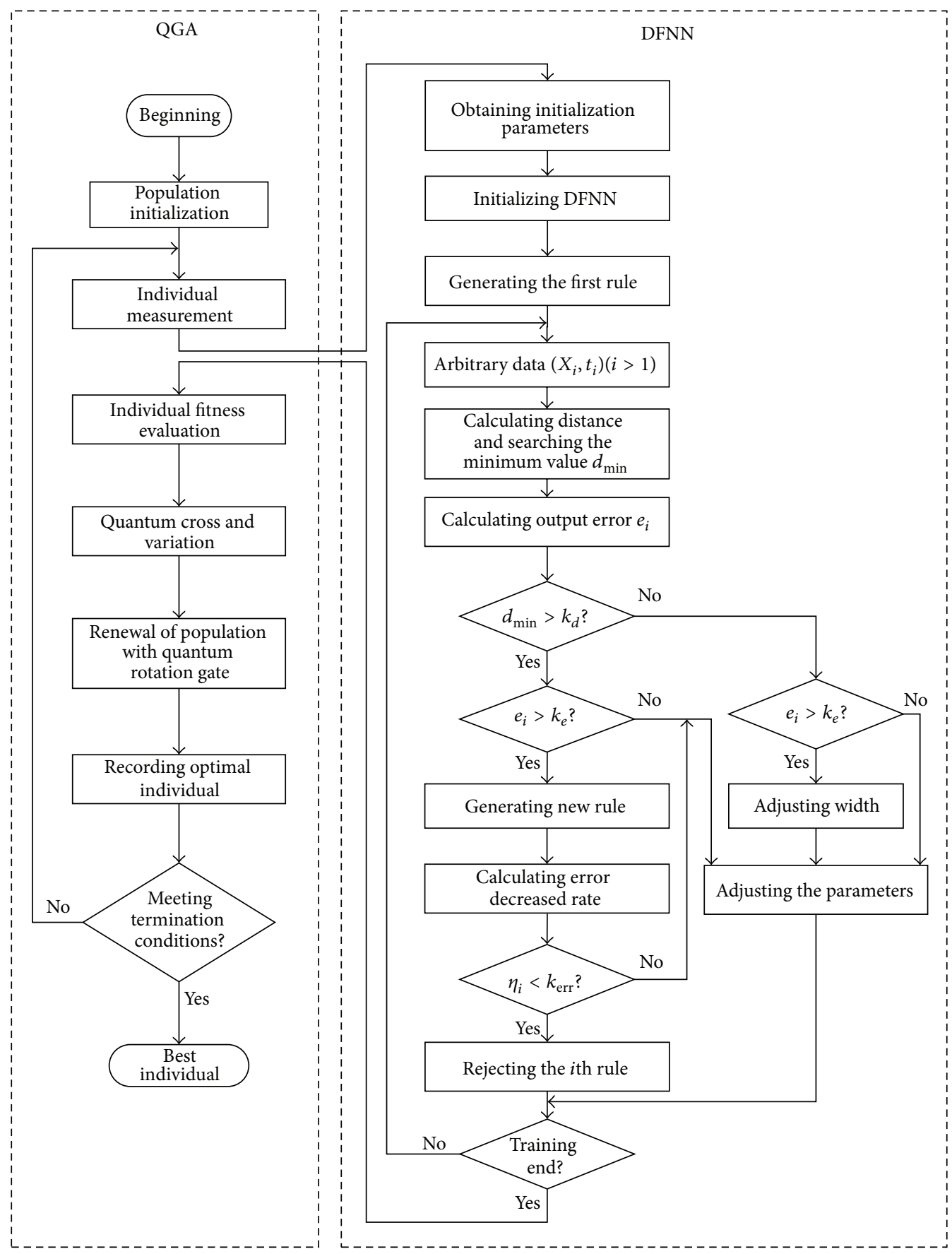

FIgURE 5: Algorithm flowchart of QGA.

where $y_{p}^{\prime}$ is the predictive value of the imitating prediction sample, $t_{p}$ is the true value of the imitating prediction sample, and $s$ is the number of the imitating prediction samples.

For DFNN training processing, the predictive values of the testing samples are prone to distortion though the predictive values of the training samples are very good. To avoid having a seriously distorted prediction, the fitness function and imitating prediction samples can be used. The fitness function takes into account the fitting degree of the training samples and the portability of optimized DFNN. Therefore, the prediction accuracy of the optimized DFNN is guaranteed.

3.2.2. Quantum Cross and Variation. In order to avoid falling into population local optimum, quantum cross has been used here. With the help of quantum cross, new chromosomes are generated which means information exchange among chromosomes is realized. The cross processes are as follows. 
TABLE 1: The adjustment strategy of the quantum rotating angle.

\begin{tabular}{cccccccc}
\hline \multirow{2}{*}{$x_{i}$} & $b_{i}$ & \multirow{2}{*}{$f(x)>f(b)$} & \multirow{2}{*}{$\Delta \theta_{i}$} & \multicolumn{5}{c}{$s\left(\alpha_{i}, \beta_{i}\right)$} \\
& & & $\alpha_{i} \beta_{i}>0$ & $\alpha_{i} \beta_{i}<0$ & $\alpha_{i}=0$ & $\beta_{i}=0$ \\
\hline 0 & 0 & TRUE/FALSE & 0 & 0 & 0 & 0 & 0 \\
0 & 1 & FALSE & $\omega$ & +1 & -1 & 0 & \pm 1 \\
0 & 1 & TRUE & $\omega$ & -1 & +1 & \pm 1 & 0 \\
1 & 0 & FALSE & $\omega$ & -1 & +1 & \pm 1 & 0 \\
1 & 0 & TRUE & $\omega$ & +1 & -1 & 0 & \pm 1 \\
1 & 1 & TRUE/FALSE & 0 & 0 & 0 & 0 & 0 \\
\hline
\end{tabular}

(1) Two chromosomes are randomly selected from the population, and whether cross operation should be considered is determined by cross probability.

(2) If it is necessary to consider the cross operation, exchanging the random cross position information between two chromosomes is applied here.

(3) Examining chromosome feasibility, cross operation is finished.

By using quantum variation, we can disturb the current evolution direction of the chromosome to avoid early maturity, so good global search capacity can be obtained.

3.2.3. Quantum Rotation Gate. Quantum gate is the actuator of the evolution process for QGA. The update process of quantum rotation gate is as follows:

$$
\left[\begin{array}{l}
\alpha^{\prime} \\
\beta^{\prime}
\end{array}\right]=\left[\begin{array}{cc}
\cos (\theta) & -\sin (\theta) \\
\sin (\theta) & \cos (\theta)
\end{array}\right]\left[\begin{array}{l}
\alpha \\
\beta
\end{array}\right],
$$

where $\left[\begin{array}{ll}\alpha & \beta\end{array}\right]^{T}$ and $\left[\begin{array}{ll}\alpha^{\prime} & \beta^{\prime}\end{array}\right]^{T}$ represent quantum bit probability amplitudes before and after updating, respectively. $\theta$ is the quantum rotating angle. The adjustment strategy of the quantum rotating angle is shown in Table 1.

Where $x_{i}$ and $b_{i}$ are the $i$ th bit of the current chromosome and the best chromosome, respectively, $\Delta \theta_{i}$ is the adjusting angle step and $s\left(\alpha_{i}, \beta_{i}\right)$ is the rotating angle direction. This paper adopts dynamic adjustment strategy based on expansion coefficient for quantum rotation gate [18-21]; rotating angle $\omega$ is defined as follows:

$$
\omega=\omega_{\min }+\left(\omega_{\max }-\omega_{\min }\right)\left[1-\left(\frac{t_{i}}{t_{\max }}\right)^{\varepsilon}\right],
$$

where $\omega_{\min }$ and $\omega_{\max }$ are the minimum value and maximum value of the $\omega$, respectively, $t_{i}$ is the number of the current genetic generations, $t_{\max }$ is the number of the maximum genetic generations, and $\varepsilon$ is the expansion coefficient.

3.3. Screw Performance Degradation Model. Sensor signals are acquired by screw online monitoring system and high dimension features are extracted in both time domain and frequency domain. Then the feature vectors can be obtained by PCA. Feature vectors are sent to feature vectors library together with the real-time working conditions. Training samples and imitating prediction samples are randomly selected from the feature vectors library and used for training
DFNN and QGA. After training, testing samples selected from the feature vectors library are used to test the optimized and trained DFNN. If the prediction accuracy falls on the system error allowable range, this DFNN can be used as screw performance degradation model. Otherwise, the DFNN must be retrained by means of increasing samples or modifying network parameters until the prediction accuracy can be guaranteed. Flowchart of building screw performance degradation model is showed in Figure 6.

\section{The Experiment Results}

Screw vibration signals are not only determined by screw performance degradation degree, but also determined by working conditions. Feature vectors library which includes real-time working conditions is accumulated with the help of the screw online monitoring system. In this paper, one ball screw of CINCINNATIV5-3000 is used as the experiment object. Four class performance degradation samples of the ball screw are randomly selected from the feature vectors library together with their working conditions. The length of each screw performance sample is 10 seconds while the sampling frequency is $2.56 \mathrm{kHz}$. Four class screw performance samples include 0.5-year-service screw samples, 2year-service screw samples, 5-year-service screw samples, and 7-year-service screw samples. Training samples include 400 samples (100 samples from each class), imitating prediction samples include 400 samples (100 samples from each class), and testing samples include 400 samples (100 samples from each class).

A mapping method used for describing the output of the screw performance degradation model is presented. In this method, different output intervals of the screw performance degradation model show different kinds of screw performance. Interval $[0,0.2)$ indicates good performance. Interval $[0.2,0.4)$ indicates the performance degraded slightly. Interval $[0.4,0.6)$ indicates middle degraded performance which can still ensure the machining accuracy. Interval $[0.6,0.8)$ presents serious degraded performance which means the screw is easy to go wrong. Interval $[0.8,1]$ means screw with failure. Combined with the field experiences, the model output of the 0.5-year-service screw samples, 2-year-service screw samples, 5-year-service screw samples, and 7-yearservice screw samples is $0.1,0.25,0.7$, and 0.9 .

The QGA population scale and the number of the iterations should be based on comprehensive consideration of training samples and searching efficiency. The parameters of the quantum cross, variation, and rotation gate can be determined by experiences on the premise of fast searching optimization. The initialization parameters range of DFNN can be obtained with multiple algorithm running tests [2225]. In this paper, parameters of the QGA are selected as follows [26, 27]: population scale is 40 , the number of the iterations is 100 , the initialization parameters interval of DFNN is $[0,3]$, cross probability is 0.3 while variation probability is 0.1 , maximum rotating angle $\omega_{\max }$ is $0.15 \pi$ and minimum rotating angle $\omega_{\min }$ is $0.01 \pi$, and expansion coefficient $\varepsilon$ is 2 . 


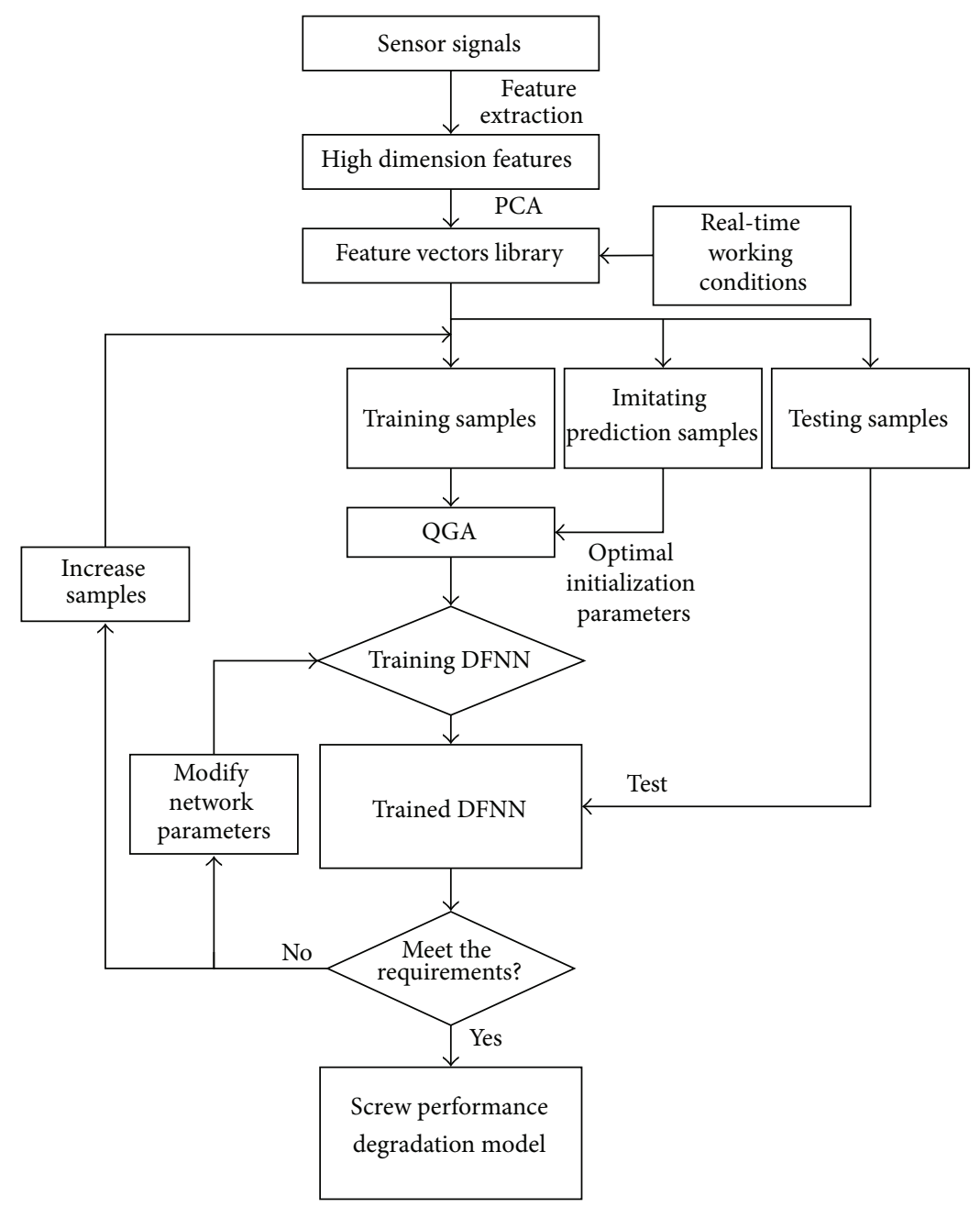

FIGURE 6: Flowchart of building screw performance degradation model.

TABLE 2: The prediction accuracies of two DFNN.

\begin{tabular}{lccccccccccccc}
\hline & $\sigma_{0}$ & $k$ & $k_{w}$ & $k_{\mathrm{err}}$ & $\gamma$ & $\beta$ & $\varepsilon_{\max }$ & $\varepsilon_{\min }$ & $e_{\max }$ & $e_{\min }$ & $\begin{array}{c}\text { Accuracy of training } \\
\text { samples }\end{array}$ & $\begin{array}{c}\text { Accuracy of testing } \\
\text { samples }\end{array}$ \\
\hline $\begin{array}{l}\text { Unoptimized } \\
\text { DFNN }\end{array}$ & 2 & 1.5 & 2 & 0.1 & 0.9 & 0.9 & 2 & 0.1 & 2 & 0.1 & 0.7350 & 0.6975 \\
\hline $\begin{array}{l}\text { Optimized } \\
\text { DFNN }\end{array}$ & 2.3896 & 1.0065 & 2.0226 & 0.0036 & 0.7432 & 0.1840 & 1.6578 & 0.3766 & 2.3861 & 0.5571 & & 0.8225 & \\
\hline
\end{tabular}

Table 2 shows the prediction accuracies of two DFNN. Compared with the unoptimized DFNN, the optimized DFNN has higher prediction accuracies in both training samples and testing samples. From the prediction accuracies of training samples and testing samples, it is clear that the optimized DFNN maintains a stable prediction accuracy.

Figures 7 and 8 show the confusion matrixes of unoptimized DFNN. It can be found that unoptimized DFNN could not distinguish 5-year-service screw very well, especially for testing samples. Figures 9 and 10 are the confusion matrixes of optimized DFNN. It can be seen that optimized DFNN shows better prediction accuracies. Figures 11 and 12 show the training and testing accumulated errors of two DFNN.
It is clear that optimized DFNN presents better prediction accuracies.

In order to validate the effect of the optimized DFNN, backpropagation (BP) neural network and radial basis function (RBF) neural network are trained and tested with the same samples. Table 3 shows the prediction accuracies of three networks. According to the results presented in Table 3, prediction accuracy of the optimized DFNN is better than that of BP neural network and RBF neural network.

Figures 13 and 14 are the confusion matrixes of $\mathrm{BP}$ neural network while Figures 15 and 16 are the confusion matrixes of RBF neural network. From Figure 13 to 16, it is found that 2-year-service screw and 5-year-service screw are 
TABLE 3: The prediction accuracies of three networks.

\begin{tabular}{lccc}
\hline & Optimized DFNN & BP neural network & RBF neural network \\
\hline Accuracy of training samples & 0.8225 & 0.6950 & 0.6925 \\
Accuracy of testing samples & 0.8175 & 0.6375 & 0.6450 \\
\hline
\end{tabular}

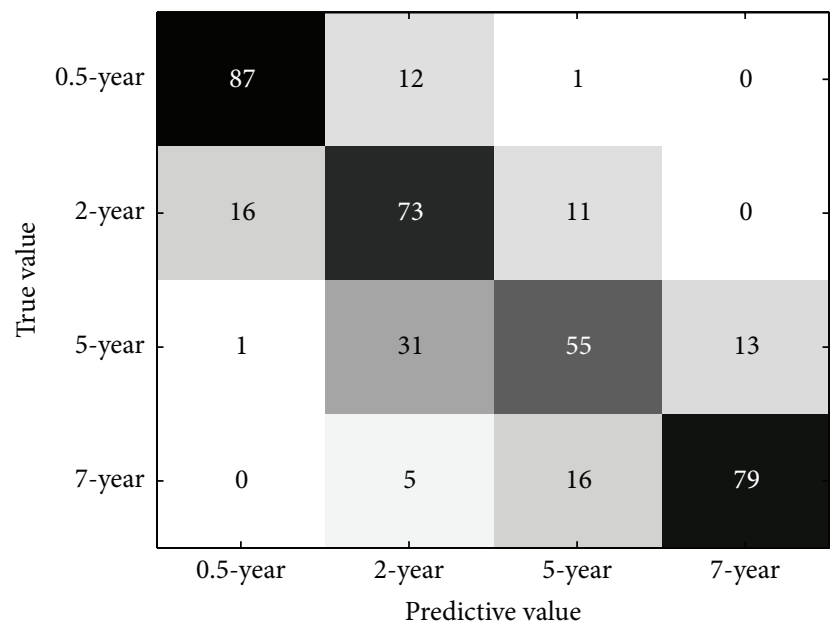

FIGURE 7: Unoptimized DFNN confusion matrix for training samples.

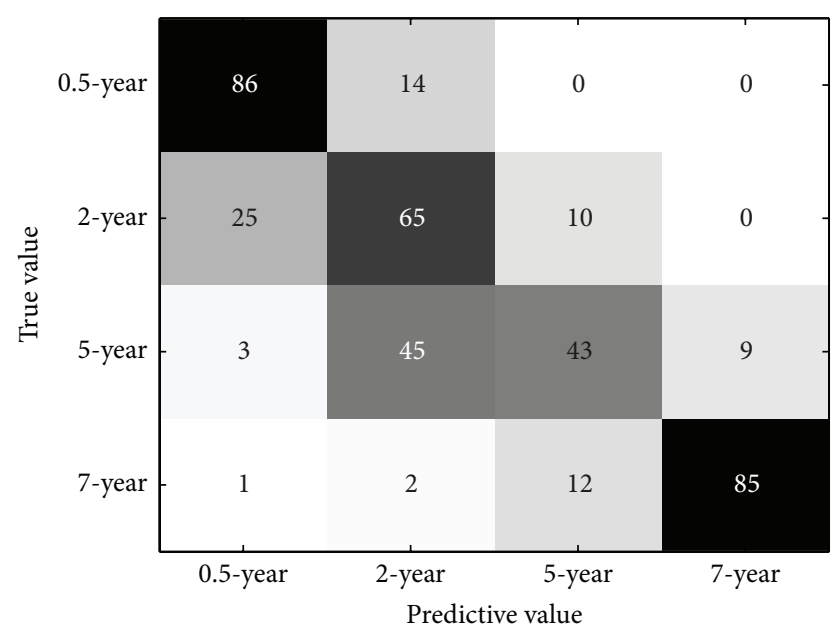

FIGURE 8: Unoptimized DFNN confusion matrix for testing samples.

difficult to distinguish both for BP neural network and for RBF neural network. It is known that screw performance degradation would obey the rule of bathtub curve; 2-yearservice screw and 5-year-service screw are in random failure period. Therefore it is not easy to predict 2-year-service screw and 5-year-service screw. Compared with Figures 9 and 10, the optimized DFNN shows better prediction accuracies than BP neural network and RBF neural network.

Figures 17 and 18 show the training and testing accumulated errors of three networks. As seen in Figures 17 and

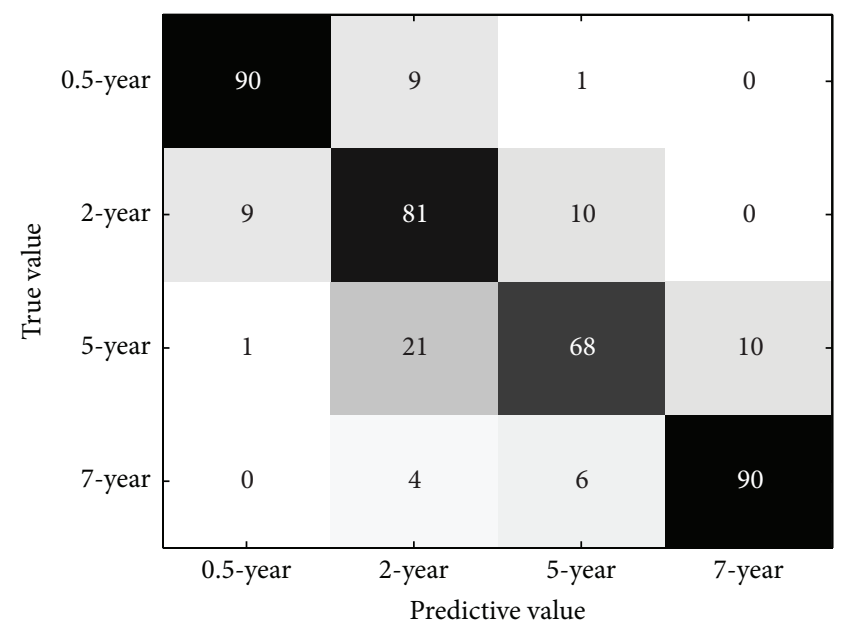

FIGURE 9: Optimized DFNN confusion matrix for training samples.

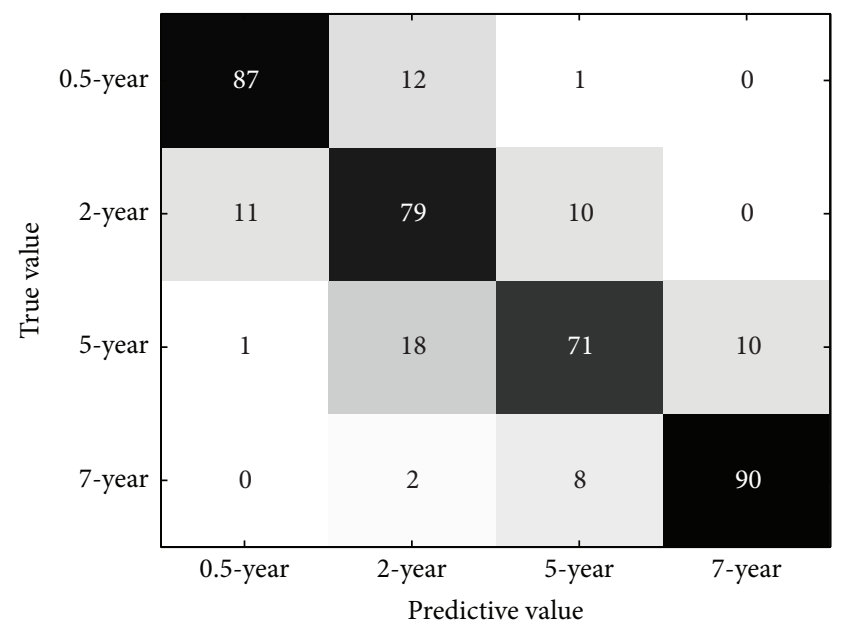

FIGURE 10: Optimized DFNN confusion matrix for testing samples.

18, the accumulated errors of BP network and RBF network are larger than those of the optimized DFNN. As previously discussed, it can be concluded that the optimized DFNN can assess screw performance degradation effectively.

\section{Conclusion}

Screw performance degradation assessment based on QGA and DFNN is studied in this paper. The experiment results classified by the optimized DFNN show that our proposed 


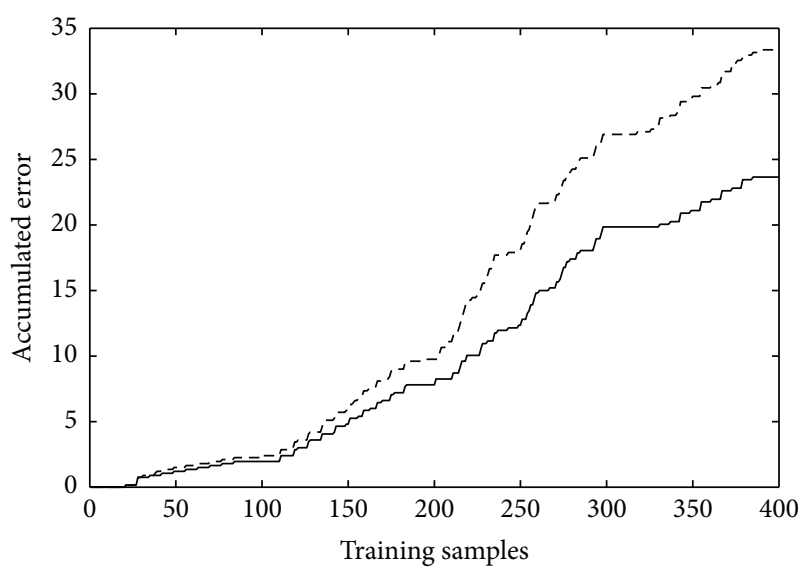

- Accumulated error of the optimized DFNN

- - - Accumulated error of the unoptimized DFNN

FIGURE 11: Training accumulated errors of two DFNN.

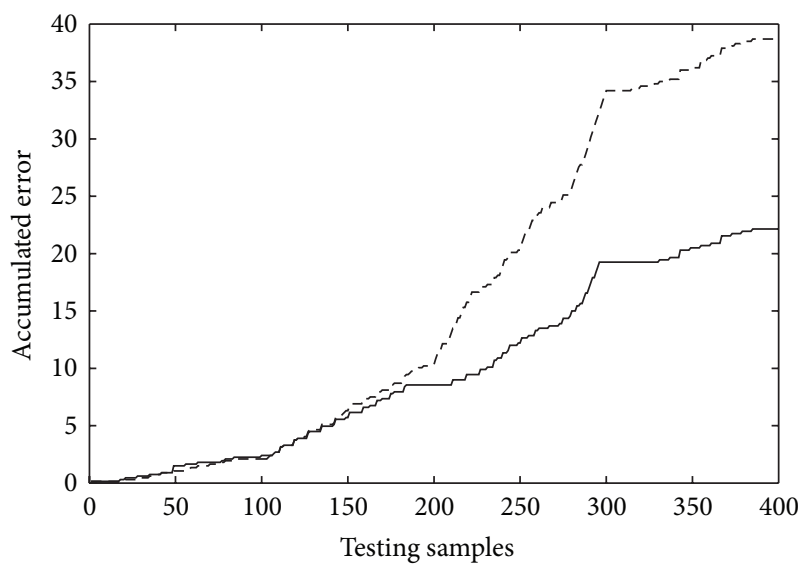

- Accumulated error of the optimized DFNN

- - - Accumulated error of the unoptimized DFNN

FIgURE 12: Testing accumulated errors of two DFNN.

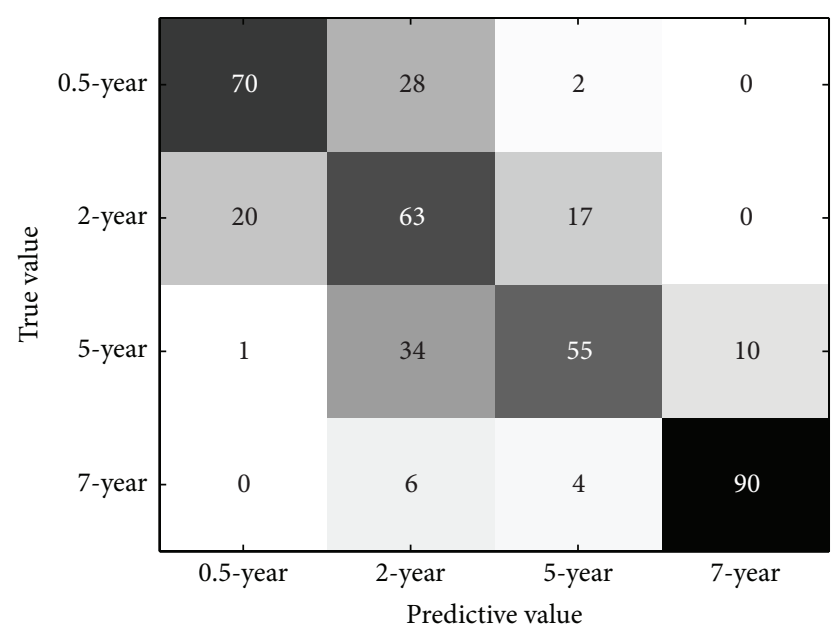

FIGURE 13: BP neural network confusion matrix for training samples.

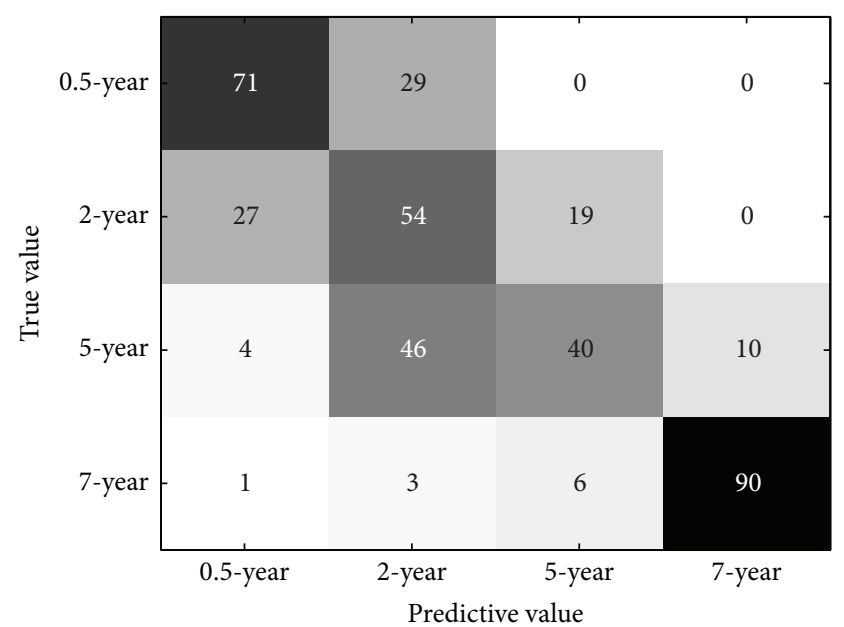

FIGURE 14: BP neural network confusion matrix for testing samples.

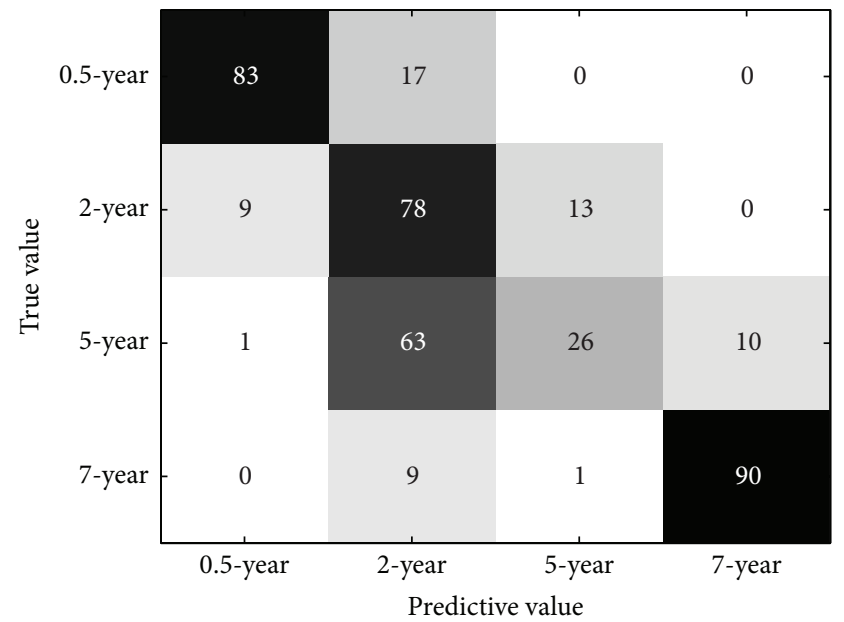

FIGURE 15: RBF neural network confusion matrix for training samples.

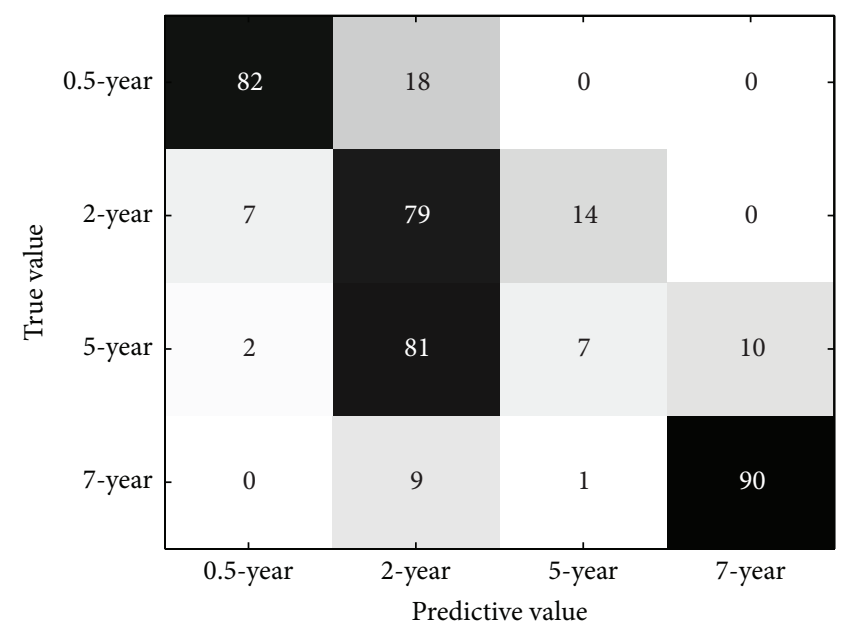

FIGURE 16: RBF neural network confusion matrix for testing samples. 


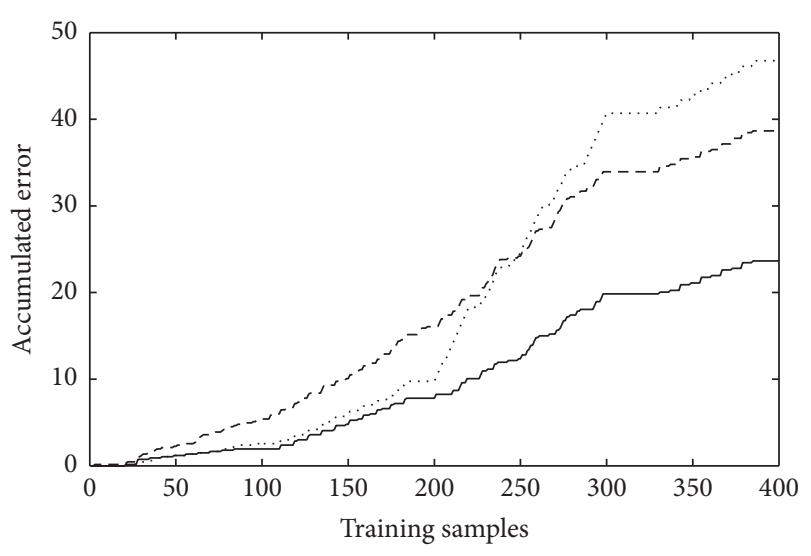

- Accumulated error of the optimized DFNN

- - - Accumulated error of BP network

..... Accumulated error of RBF network

FIgURE 17: Training accumulated errors of three networks.

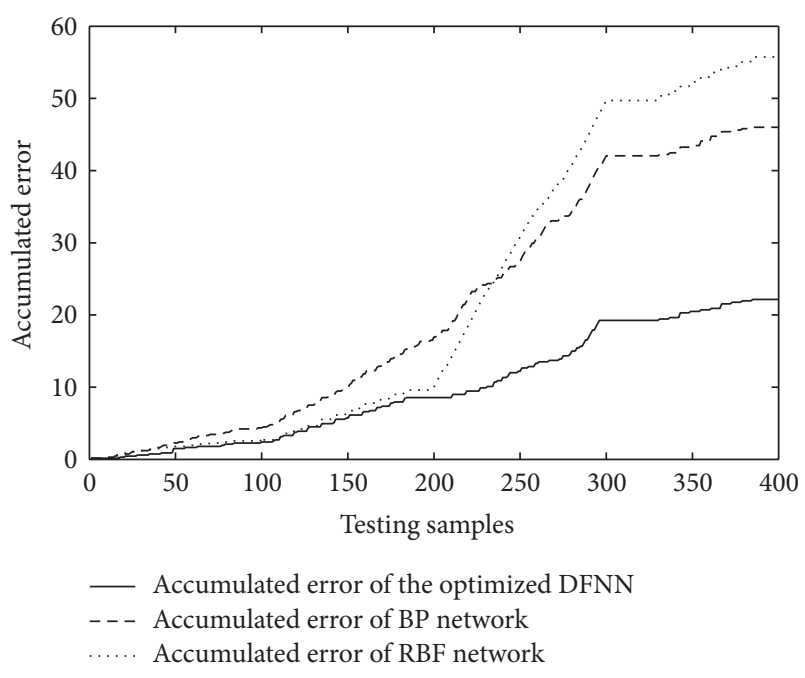

FIGURE 18: Testing accumulated errors of three networks.

method has the best performance compared to other three models.

(1) Screw online monitoring system is applied to CINCINNATIV5-3000 machining center. Considering that the machining center always works on changeable working conditions, the real-time working conditions are also stored as an important part of the feature vectors library.

(2) To solve the prediction stability problem of using DFNN model, an optimization algorithm based on QGA and imitating prediction samples is presented in this paper. Compared to the unoptimized DFNN, the optimized DFNN maintains a good prediction accuracy.

(3) Screw feature vectors of different service life are applied to test the model performance in the experiment; from the discussion among three network models, the conclusion can be reached that the optimized DFNN presents better prediction accuracy than BP and RBF network and is suitable for screw performance assessment.

\section{Conflict of Interests}

The authors declare that there is no conflict of interests regarding the publication of this paper.

\section{Acknowledgment}

The authors would like to extend their thanks to the joint financial support by National Natural Science Fund of China (51275426).

\section{References}

[1] C.-H. Lee, M.-Y. Yang, C.-W. Oh, T.-W. Gim, and J.-Y. Ha, “An integrated prediction model including the cutting process for virtual product development of machine tools," International Journal of Machine Tools and Manufacture, vol. 90, pp. 29-43, 2015.

[2] R. Pérez, A. Molina, and M. Ramírez-Cadena, "Development of an integrated approach to the design of reconfigurable micro/mesoscale cnc machine tools," Journal of Manufacturing Science and Engineering, vol. 136, no. 3, Article ID 031003, 10 pages, 2014.

[3] A. Gok, C. Gologlu, and H. I. Demirci, "Cutting parameter and tool path style effects on cutting force and tool deflection in machining of convex and concave inclined surfaces," International Journal of Advanced Manufacturing Technology, vol. 69, no. 5-8, pp. 1063-1078, 2013.

[4] A. Deshpande, "An empirical study to evaluate machine tool production readiness and performance," International Journal of Advanced Manufacturing Technology, vol. 64, no. 9-12, pp. 1285-1296, 2013

[5] P. C. Tsai, C. C. Cheng, and Y. C. Hwang, "Ball screw preload loss detection using ball pass frequency," Mechanical Systems and Signal Processing, vol. 48, no. 1-2, pp. 77-91, 2014.

[6] Z. H. Li, K. G. Fan, J. G. Yang, and Y. Zhang, "Timevarying positioning error modeling and compensation for ball screw systems based on simulation and experimental analysis," International Journal of Advanced Manufacturing Technology, vol. 73, no. 5-8, pp. 773-782, 2014.

[7] Y. Pan, M. J. Er, X. Li, H. Yu, and R. Gouriveau, "Machine health condition prediction via online dynamic fuzzy neural networks," Engineering Applications of Artificial Intelligence, vol. 35, no. 1, pp. 105-113, 2014.

[8] J. Mateo, A. M. Torres, and M. A. García, "Dynamic fuzzy neural network based learning algorithms for ocular artefact reduction in EEG recordings," Neural Processing Letters, vol. 39, no. 1, pp. 45-67, 2014.

[9] C.-F. Juang, "A TSK-type recurrent fuzzy network for dynamic systems processing by neural network and genetic algorithms," IEEE Transactions on Fuzzy Systems, vol. 10, no. 2, pp. 155-170, 2002.

[10] C.-H. Lee and C.-C. Teng, "Identification and control of dynamic systems using recurrent fuzzy neural networks," IEEE Transactions on Fuzzy Systems, vol. 8, no. 4, pp. 349-366, 2000. 
[11] R.-J. Wai and P.-C. Chen, "Robust neural-fuzzy-network control for robot manipulator including actuator dynamics," IEEE Transactions on Industrial Electronics, vol. 53, no. 4, pp. 13281349, 2006.

[12] A. Subasi, "Automatic detection of epileptic seizure using dynamic fuzzy neural networks," Expert Systems with Applications, vol. 31, no. 2, pp. 320-328, 2006.

[13] S. Q. Wu, M. J. Er, and Y. Gao, "A fast approach for automatic generation of fuzzy rules by generalized dynamic fuzzy neural networks," IEEE Transactions on Fuzzy Systems, vol. 9, no. 4, pp. 578-594, 2001.

[14] H. Adeli and X. M. Jiang, "Dynamic fuzzy wavelet neural network model for structural system identification," Journal of Structural Engineering, vol. 132, no. 1, pp. 102-111, 2006.

[15] C.-F. Juang and C.-D. Hsieh, "A locally recurrent fuzzy neural network with support vector regression for dynamic-system modeling," IEEE Transactions on Fuzzy Systems, vol. 18, no. 2, pp. 261-273, 2010.

[16] C. G. Huang, G. Li, Z. X. Xu, and L. P. Chang, "Design of optimal digital lattice filter structures based on genetic algorithm," Signal Processing, vol. 92, no. 4, pp. 989-998, 2012.

[17] L. Liu, L.-H. Guo, H. Xiao, J.-J. Wang, and G.-G. Wang, "Software reliability growth model based on SAA-DFNN," Journal of Jilin University, vol. 42, no. 5, pp. 1225-1230, 2012.

[18] X. C. Zhang, H. L. Gao, H. F. Huang, L. Guo, and S. D. Xiao, "Optimization design of mathematical morphology filter based on quantum genetic algorithm," Journal of Southwest Jiaotong University, vol. 49, no. 3, pp. 462-469, 2014.

[19] K.-H. Han and J.-H. Kim, "Quantum-inspired evolutionary algorithm for a class of combinatorial optimization," IEEE Transactions on Evolutionary Computation, vol. 6, no. 6, pp. 580-593, 2002.

[20] J. Zhang, H. Z. Li, Z. G. Tang, Q. P. Lu, X. O. Zheng, and J. L. Zhou, "An improved quantum-inspired genetic algorithm for image multilevel thresholding segmentation," Mathematical Problems in Engineering, vol. 2014, Article ID 295402, 12 pages, 2014.

[21] S. E.-O. Bahlous, M. Neifar, S. El-Borgi, and H. Smaoui, "Ambient vibration based damage diagnosis using statistical modal filtering and genetic algorithm: a bridge case study," Shock and Vibration, vol. 20, no. 1, pp. 181-188, 2013.

[22] A. Montazeri and J. Poshtan, "Optimizing a multi-channel ANC system for broadband noise cancellation in a telephone kiosk using genetic algorithms," Shock and Vibration, vol. 16, no. 3, pp. 241-260, 2009.

[23] A. Lin and J. Phillips, "Optimization of random diffraction gratings in thin-film solar cells using genetic algorithms," Solar Energy Materials and Solar Cells, vol. 92, no. 12, pp. 1689-1696, 2008.

[24] J.-C. Lee, W.-M. Lin, G.-C. Liao, and T.-P. Tsao, "Quantum genetic algorithm for dynamic economic dispatch with valvepoint effects and including wind power system," International Journal of Electrical Power and Energy Systems, vol. 33, no. 2, pp. 189-197, 2011.

[25] M. J. Er, F. Liu, and M. B. Li, "Channel equalization using dynamic fuzzy neural networks," International Journal of Fuzzy Systems, vol. 11, no. 1, pp. 10-19, 2009.

[26] A. SaiToh, R. Rahimi, and M. Nakahara, "A quantum genetic algorithm with quantum crossover and mutation operations," Quantum Information Processing, vol. 13, no. 3, pp. 737-755, 2014.
[27] J. W. Gu, M. Z. Gu, C. W. Cao, and X. S. Gu, "A novel competitive co-evolutionary quantum genetic algorithm for stochastic job shop scheduling problem," Computers \& Operations Research, vol. 37, no. 5, pp. 927-937, 2010. 

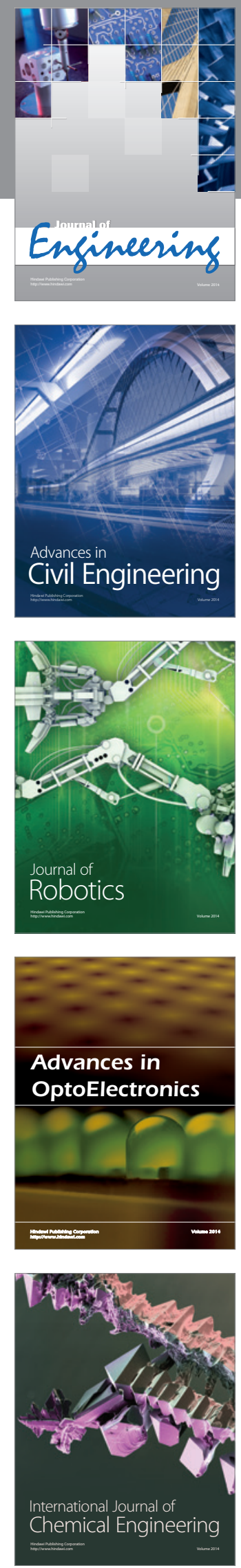

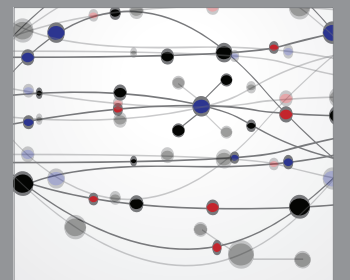

The Scientific World Journal
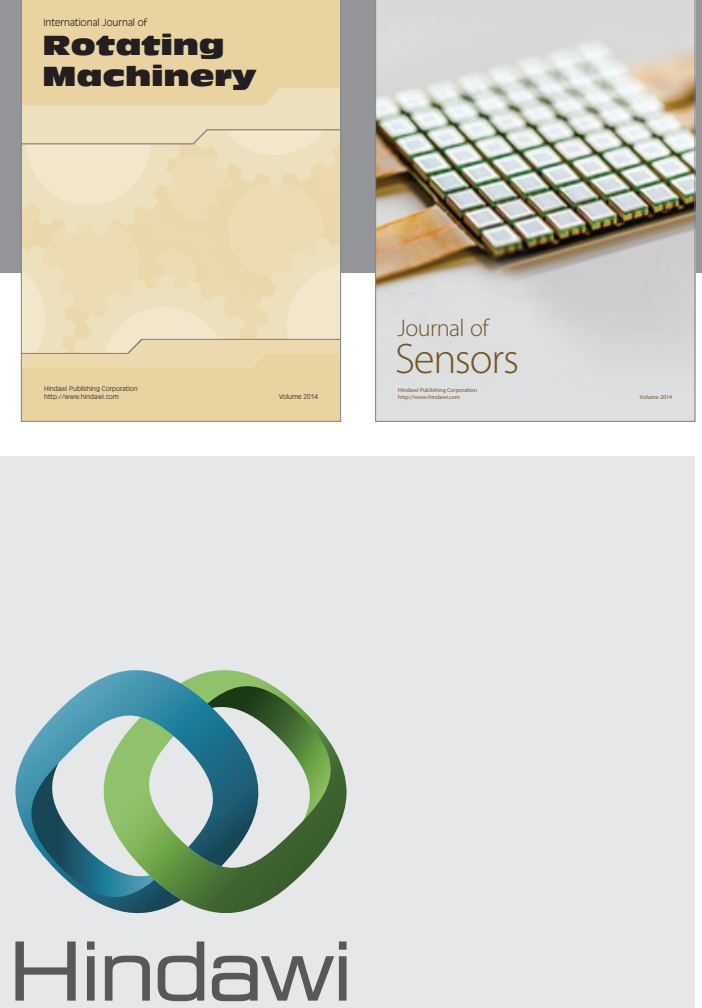

Submit your manuscripts at http://www.hindawi.com
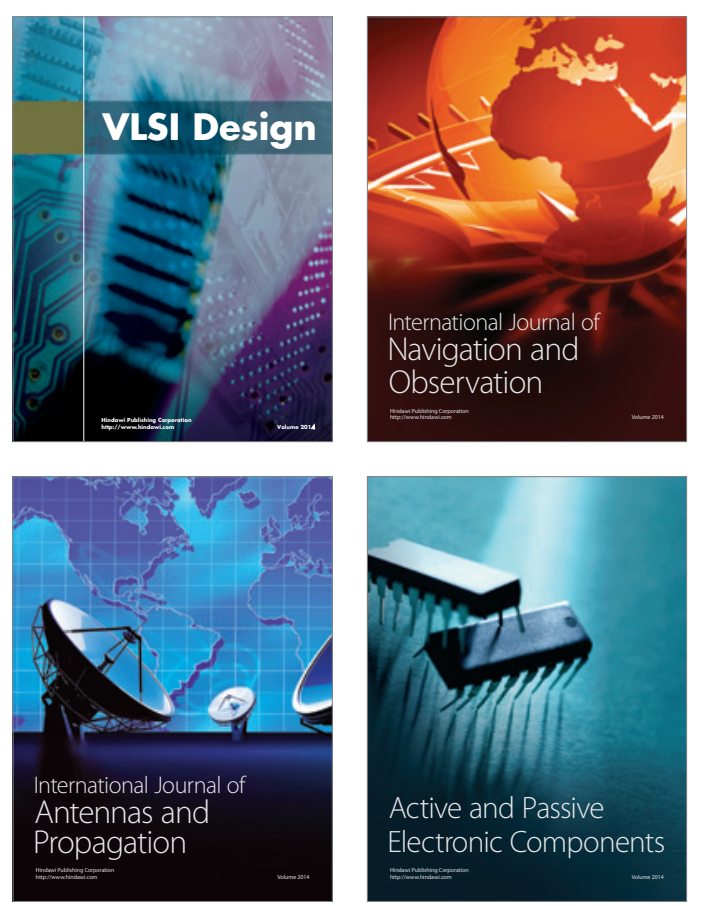
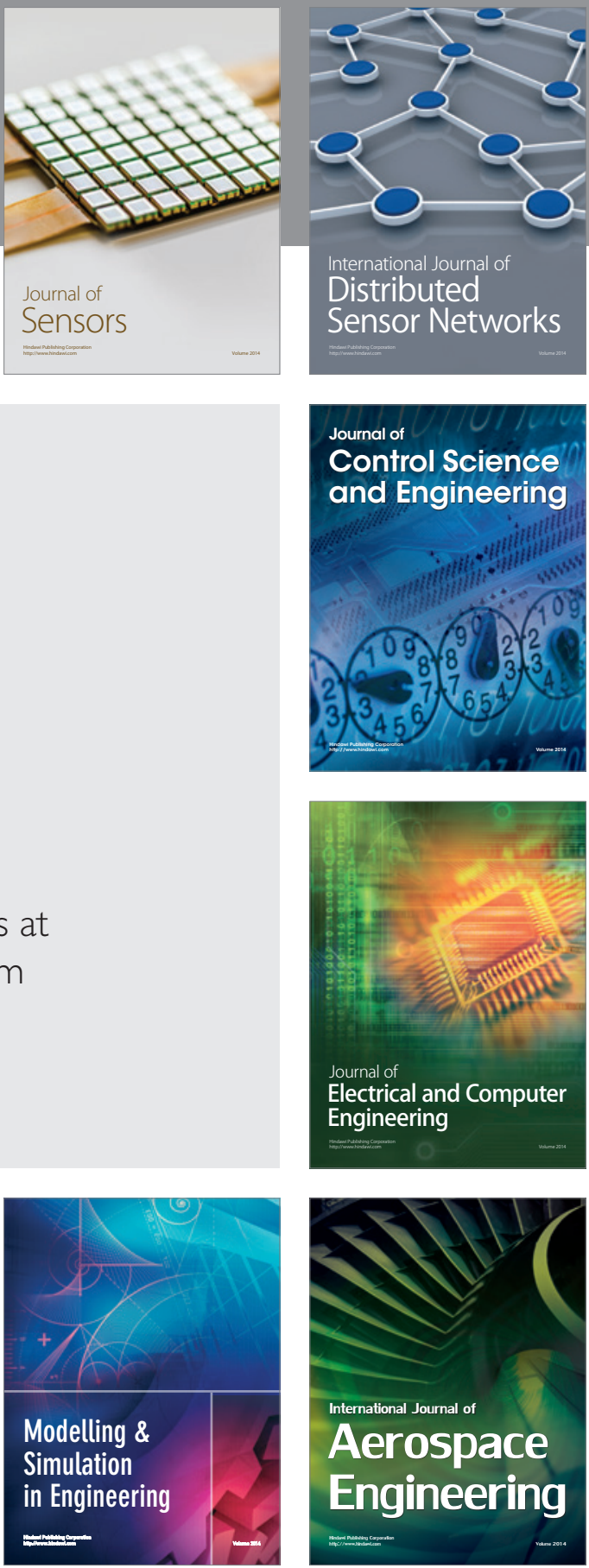

Journal of

Control Science

and Engineering
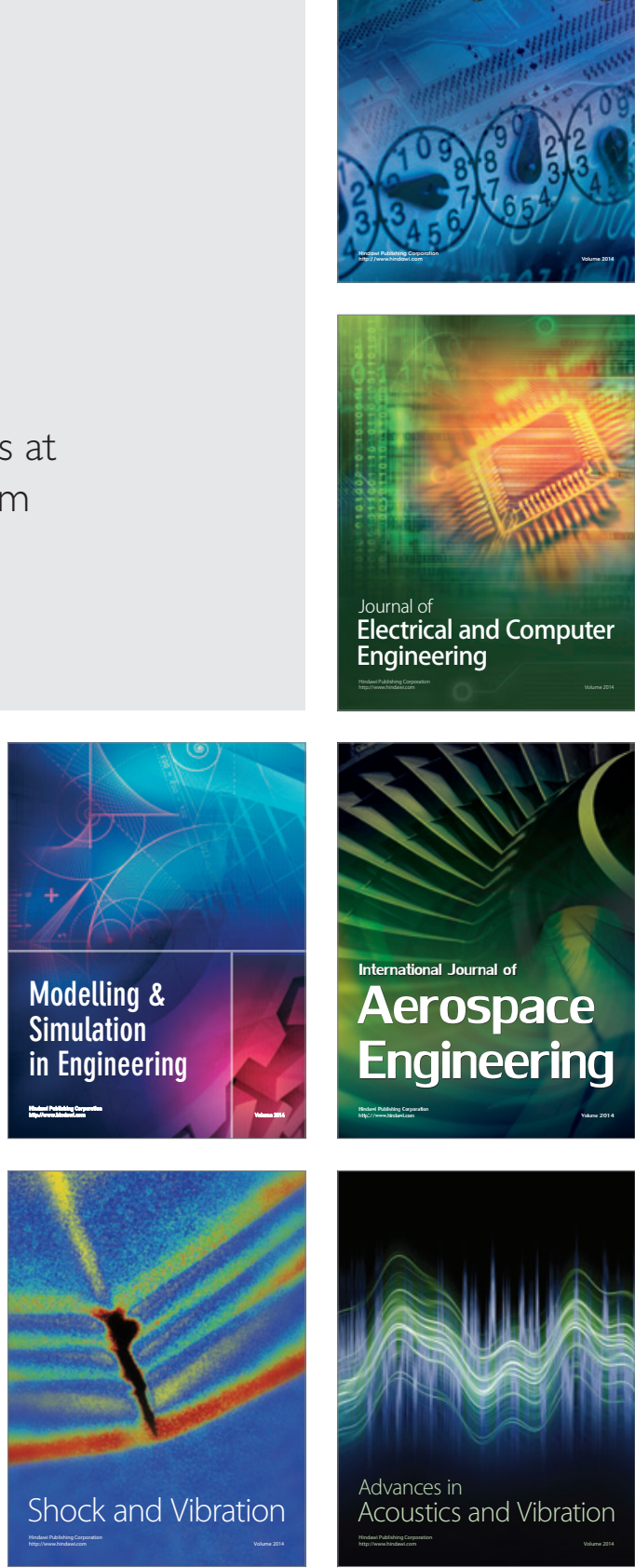